\title{
Ray Tracing Methods for Correcting Chromatic Aberrations in Imaging Systems
}

\author{
Dmitry Reshidko, ${ }^{1}$ Masatsugu Nakanato, ${ }^{2}$ and José Sasián ${ }^{1}$ \\ ${ }^{1}$ College of Optical Sciences, University of Arizona, Tucson, AZ 85721, USA \\ ${ }^{2}$ Canon USA, 9030 South Rita Road, Suite 302, Tucson, AZ 85747, USA \\ Correspondence should be addressed to Dmitry Reshidko; dmitry@optics.arizona.edu
}

Received 26 November 2013; Accepted 18 February 2014; Published 30 March 2014

Academic Editor: Augusto Beléndez

Copyright (c) 2014 Dmitry Reshidko et al. This is an open access article distributed under the Creative Commons Attribution License, which permits unrestricted use, distribution, and reproduction in any medium, provided the original work is properly cited.

The correction of chromatic aberrations is typically performed using aberration formulas or by using real ray tracing. While the use of aberration formulas might be effective for some simple optical systems, it has limitations for complex and fast systems. For this reason chromatic aberration correction is usually accomplished with real ray tracing. However, existing optimization tools in lens design software typically mix the correction of monochromatic and chromatic aberrations by construction of an error function that minimizes both aberrations at the same time. This mixing makes the correction of one aberration type dependent on the correction of the other aberration type. We show two methods to separate the chromatic aberrations correction of a lens system. In the first method we use forward and reverse ray tracing and fictitious nondispersive glasses, to cancel the monochromatic aberration content and allow the ray tracing optimization to focus mainly on the color correction. On the second method we provide the algorithm for an error function that separates aberrations. Furthermore, we also demonstrate how these ray tracing methods can be applied to athermalize an optical system. We are unaware that these simple but effective methods have been already discussed in detail by other authors.

\section{Introduction}

Correction of chromatic aberrations in imaging systems has been the subject of research for many generations of scientists and still remains one of the most challenging problems in lens design. The early experiments of Isaac Newton, which later were developed into his color theory, started a detailed understanding of chromatic effects in optical systems. Since Conrady's solution to the powers for an apochromatic triplet that uses the partial dispersion versus $V$-number graph, many graphical methods have been devised for glass selection. In particular Hoogland's method is considered the best according to Rayces and Rosete-Aguilar [1,2]. More comprehensive glass selection methods have been also developed for finding proper glass combinations to design systems corrected for two, three, four, and even more wavelengths. A notable example is the method of Robb and Mercado $[3,4]$ that is based on Buchdahl chromatic coordinates. This work has been extended by Albuquerque et al. [5] to include higher order aberration and to include some minimization metrics. Sigler [6] points out that chromatic aberration correction through the selection of optical glass has been one of the most extensively studied subjects in the field of lens design.

While excellent results for color correction are reported in the literature, there is still a gap between theory and practice and many methods can be considered purely academic. Aberration formulas are typically based on the first order properties of the system or include only final number of higher order aberration coefficients. However, the complexity of actual systems grows and simple metrics are not sufficient to estimate the final chromatic performance. Lens designers deal with folded, multireflection systems, nonsymmetrical designs, and compact packaging. Chromatic aberration correction methods should not only be capable of finding solutions for simple optical systems but also be able to support correction in complex systems. Some reported techniques require additional mathematical calculation which cannot be implemented directly in the lens design software and require 
external tools. The use of additional software interrupts the lens designer work flow and requires a significant amount of time and effort.

The correction of chromatic aberrations still remains challenging in high aperture large field systems with a broad band. Modern optical instruments, such as those used in medicine, astronomy, semiconductor, defense, and security markets, take advantage of different spectral bands spanning from short UV to long IR. Depending on the application, these systems are sometimes designed to support several spectral bands. The new generation of sensors which are sensitive to a larger bandwidth, for example, from visible to SWIR, is already available commercially. In addition, some state-of-the-art applications may use two sensors supporting different bandwidths within the same optical channel.

The physics of wave propagation limit the monochromatic performance of the free-space optical system by diffraction. Chromatic aberrations add another fundamental limitation to optical systems. Modern computers have usually enough computation power to perform aberration correction with real ray tracing. Although real ray tracing optimization is time demanding, it allows us to control all desired parameters of the system.

Real ray tracing optimization is performed by minimization of some error function. However, the standard error function in lens design software typically mixes the correction of monochromatic and chromatic aberrations. In that case correction of chromatic aberration is strongly dependent on the correction of the monochromatic aberration.

In this paper we separate the chromatic aberrations correction from the correction of monochromatic aberrations of a lens system and present two methods. These methods can be implemented within optical design software and can be used to correct both chromatic change of focus and chromatic change of magnification of imaging systems for any spectral band.

In Section 2 we explain the first method of forward and reverse ray tracing and discuss its limitations. In Section 3 we explain the second method which is an algorithm for writing an error function that separates aberrations. Some examples and guidelines to application of the methods are provided in Section 4. In Section 5 we introduce a modification to the second method to aid in the athermalization of imaging systems. Section 6 concludes the paper.

\section{Forward and Reverse Ray Tracing}

In this section we present the method of forward and reversed ray tracing. One of the fundamental axioms of geometrical optics is the principle of reversibility of the optical path of rays $[7,8]$. According to this principle any ray traced through an optical system, if reversed in propagation direction, will retrace the same path backwards. Consequently, for any arbitrary optical system, all rays traced forward and then backwards will have the same optical path; that is, both chromatic and monochromatic aberrations cancel out.

To implement the method in sequential lens design software, we introduce a virtual mirror at the image plane

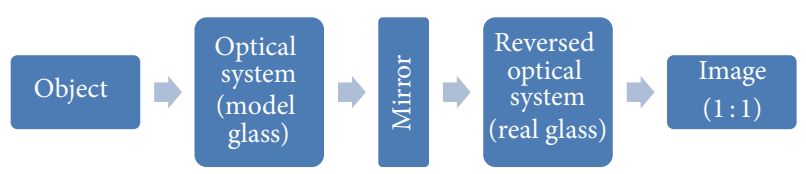

FiguRE 1: Forward and reversed ray tracing flow.

of the original optical system and add a reversed copy of the system. Note that, in our case, in contrast to regular reflection from the mirror, the sign of thicknesses remains the same. We call the part of the system before the mirror-the real part and the part after the mirror-the virtual part. This sequential model allows us to keep the same corresponding angles for the ray propagating in the real and virtual part of the system. In addition, for any ray, optical path in the real and virtual part will have the same value, but opposite sign; that is, both chromatic and monochromatic aberrations cancel out as desired. The schematic representation of the ray tracing flow is shown in Figure 1.

In order to make the chromatic aberrations show, we change the optical glasses in the real part of the system to fictitious, nondispersive model glasses. For the model glass we use the refractive index of the original glass at the primary wavelength of the design and specify zero dispersion, that is, zero glass $V$-number, and zero partial dispersion. In the real part of the system, rays that have the same object and pupil coordinates but different wavelengths will follow the same path. However, in the virtual part, the optical path will be different due to dispersion of the actual glasses. With this real and virtual lens model, for a given object point, the optical path difference will be zero for the primary wavelength due to cancellation. For other wavelengths the chromatic aberration will show because there is no cancellation between the real and virtual parts. Thus the chromatic aberration content is separated from the monochromatic aberration content.

Since only the chromatic aberration is present, the full power of an optimizer can be dedicated for color correction. This results in an advantage. We use global optimization together with iterative substitution of similar glass types to reduce the chromatic aberration through the selection of optical glass in the virtual part of the system. The real part of the system remains unchanged which will force the monochromatic solution to be close to the original design. We found that during optimization we need to control the focal length and $f / \#$ of the virtual system. In addition, the forward and reverse ray tracing model should have unit magnification. If original design was for infinite conjugate, we use a paraxial lens with focal length equal to the focal length of the original system to focus the beam.

An example of forward and reversed ray tracing model for an Edmund Optics $f=20 \mathrm{~mm}$ achromatic doublet is shown in Figure 2. The primary wavelength is the $d$ line. Both the chromatic and the monochromatic aberrations are present for the on axis field in the original design. We construct forward and reversed ray tracing model according to the procedure described above. Since the doublet is used for an infinite conjugate, we add at the end an $f=20 \mathrm{~mm}$ 


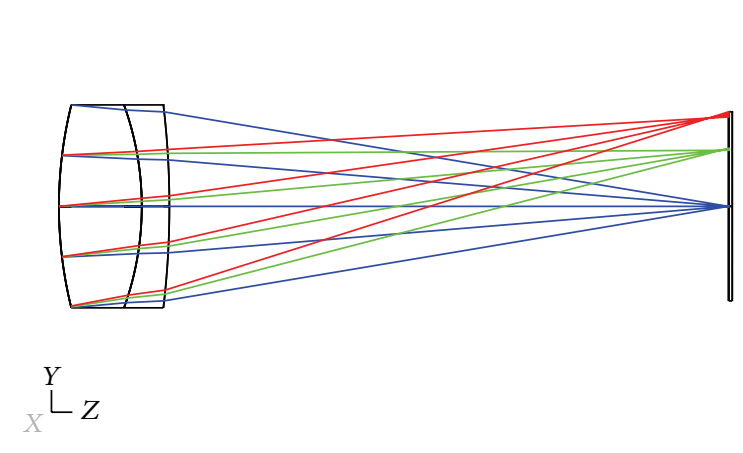

(a)

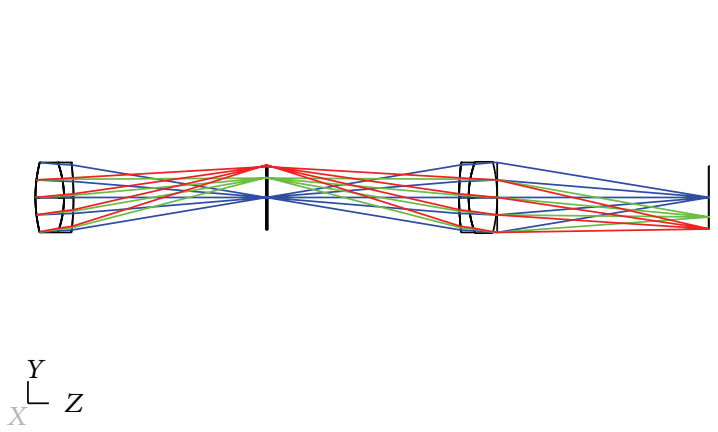

$\stackrel{Y}{L} Z$

(c)

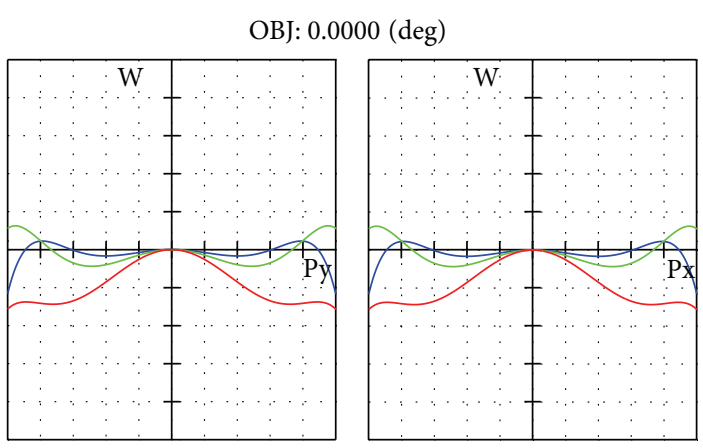

(b)

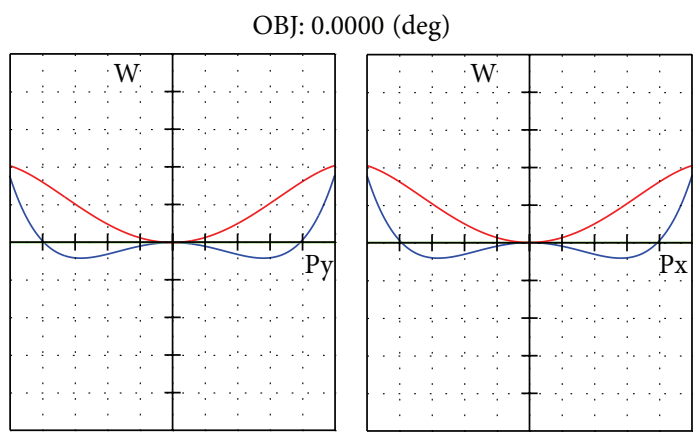

(d)

FIGURE 2: Edmund Optics $20 \mathrm{~mm}$ achromatic doublet: (a) original lens and (b) on axis OPD fan, (c) forward and reversed ray tracing model and $(\mathrm{d})$ on axis OPD fan. The OPD fan full scale is 0.5 waves.

paraxial lens to the modified system to focus the beam. Note in Figure 2(d) that for the d line the OPD (optical path difference) is zero across the aperture. All the monochromatic aberrations are canceled and only the chromatic aberrations for the $F$ and $C$ lines are present in the forward and reversed model system. This is the starting point for the optimization process.

This forward and reversed ray tracing model demonstrates the geometrical method of separation of chromatic and monochromatic aberrations. However, this method has some limitations and drawbacks. The model glass, used in the real part of the system, changes propagation of the rays for the wavelength that differ from the primary wavelength. The deviations of these rays from the original path accumulate as the rays propagate through real and virtual parts of the system. In certain cases it may cause total internal reflection, vignetting of some of the rays, or singularities in the optical path for particular object and pupil coordinates. Moreover, the model has twice as many optical surfaces as the original design, which significantly slows down ray tracing time. Nevertheless it is a useful method.

\section{Aberration Subtraction Method}

In this section we separate the chromatic and monochromatic aberrations directly in the error function. We simply subtract the aberration of the primary wavelength from the aberration due to other wavelengths.

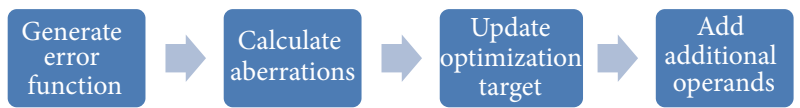

FIGURE 3: Aberration subtraction method.

In lens design software we can choose to minimize the wave aberrations or the ray aberrations. We write an error function where the optimization targets are changed as follows. For any wavelength $\lambda$ and primary wavelength $\lambda_{\text {prime }}$ we modify the wave aberration target to be

$$
\frac{\left(\mathrm{OPD}_{\lambda} \times \lambda-\mathrm{OPD}_{\text {primary }} \times \lambda_{\text {primary }}\right)}{\lambda} .
$$

For ray aberrations we subtract the primary wavelength directly without applying any normalization. Then we modify the optimization target for every field point and every pupil coordinate in the error function. The chief ray at the primary wavelength is used as a reference for both ray and wave aberrations calculations. This procedure can easily be implemented by writing a macroprogram. Figure 3 shows the aberration subtraction procedure.

We now comment on the dynamics of minimization of the modified error function. In the original design the primary wavelength's monochromatic aberration will match the target aberration value, by construction. Initially, and similarly to the forward and reversed ray tracing technique, the primary wavelength aberration will not contribute to the 
error function. During the optimization, the original lens will change and the primary wavelength's monochromatic aberration will be different. The weight placed on the primary wavelength, in the modified error function, will force the monochromatic solution to be close to the original design. Further minimization of the modified error function together with iterative substitution of similar glass types will reduce, if proper glass combinations exist, all chromatic aberrations to the same monochromatic aberration value.

The direct subtraction of the aberrations in the error function is a similar concept to the previous method of forward and reverse ray tracing. The chromatic aberrations are separated from the monochromatic aberration and minimized to the same value. While the method of Section 2 has clear geometrical model, the aberration subtraction method of this section has several noticeable advantages. First, the method is simpler: there is no need to build a complicated sequential model. Ray tracing time will not significantly be increased. Second, rays are traced only forward and there will be no issues with TIR and vignetting as discussed in the previous section. Finally, we can control the actual amount of aberration in the design. Other optimization operands can be added to the modified error function to allow better control of various design parameters. For example, if the design requirements are on the MTF, we may choose to add MTF operands.

\section{Guidelines and Example of Application of the Methods}

We emphasize that the presented methods are not aimed at improving the monochromatic performance of a system but at reducing chromatic aberration to the monochromatic value. Both presented methods could be applied at the intermediate design stage, when the monochromatic performance is close to the requirements and the chromatic aberrations are corrected to some extent. In order to gradually reduce the overall amount of aberrations in the system, the methods could be applied in an iterative process. Each iteration includes the following steps.

(i) Optimize the lens with a given error function. During this optimization one may choose to put additional weight on the primary wavelength. Putting additional weight on the primary wavelength will improve the monochromatic performance and allow a better target for application of the methods in the next step.

(ii) Apply the forward and reversed ray tracing method or aberration subtraction method. Use global optimization with iterative glass substitution.

(iii) Proceed to the next iteration till no new combinations of glass are found in a reasonable amount of time.

The flow chart in Figure 4 summarizes the algorithm.

As an example we apply the presented methods to a Canon telephoto lens [9]. This is a long-focal-length $(300 \mathrm{~mm})$ optical system that operates at $f / 2.9$. Focusing is performed by moving one of the lens units. The lens operates in the visible band from $436 \mathrm{~nm}$ to $656 \mathrm{~nm}$. The primary wavelength of the design which is also the reference for the monochromatic aberrations is $588 \mathrm{~nm}$. An original patent data for infinite and 2-meter object distance configurations is shown in Figure 5.

Our goal was to improve the polychromatic MTF while keeping the same number of elements, vignetting, focal length, and $f / \#$. We used the iteration algorithm described above. We performed three iterations with aberration subtraction method for chromatic aberration correction. In the last iteration MTF operands were added to the error function to target the polychromatic MTF. The OPD fans of the original design and iterations 1,2 , and 3 for the infinite and 2 -meter object distance configurations are shown in Figures 6 and 7. The glass combinations used in the original design and iterations 1, 2, and 3 are given in the Appendix.

In each iteration the chromatic aberration is made to match better the monochromatic aberration value. Between the iterations the system was reoptimized to minimize the monochromatic aberration. Overall, the amount of aberrations in the system is gradually reduced. The polychromatic MTF improvement is also shown in Figures 8 and 9.

This significant improvement of MTF over the entire field of view and a good balance was obtained for both the infinite and the 2-meter object distances.

\section{Aberration Subtraction Method for Athermalization of Imaging Systems}

Thermal stability is another key requirement for many lens systems. Optical systems can be exposed to a wide range of temperatures. A temperature change has the major effects of changing the index of refraction of the optical glass and the geometry of the lens elements. First-order imaging properties of the lens are affected and additional aberrations are induced due to perturbation of the system from the nominal design.

Typically a lens is designed for some mean temperature. Once a nominal design is finished, a set of glasses that satisfies both monochromatic and chromatic aberration correction requirements has been found. Such design may fail to maintain desirable image quality over the whole range of possible temperatures. In order to insure reasonable performance, the effect of a uniform, homogeneous temperature change is simulated. Athermalization, the condition of not varying with temperature, is achieved, similarly to chromatic aberration correction, by selection of materials. The goal is to keep the aberration correction invariant over the whole required temperature range. We modify the aberration subtraction method described in Section 3 to separate the aberrations induced by thermal effects from aberrations in the nominal design.

In lens design software a uniform temperature change can be modeled by adding lens configurations. Each configuration simulates some particular temperature by adjusting the index of refraction of the optical materials. A separate error function is generated for each configuration. In our method we simply subtract the aberration of the nominal configuration from the aberration of other configurations. Then we write an error function where the optimization 


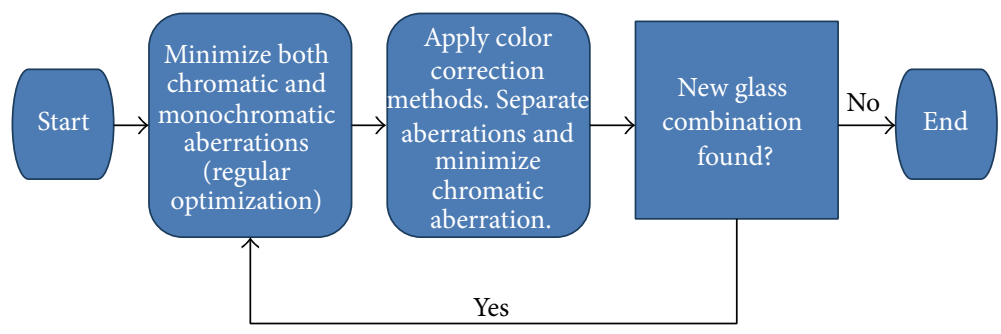

FIGURE 4: Suggested algorithm for application of presented methods.

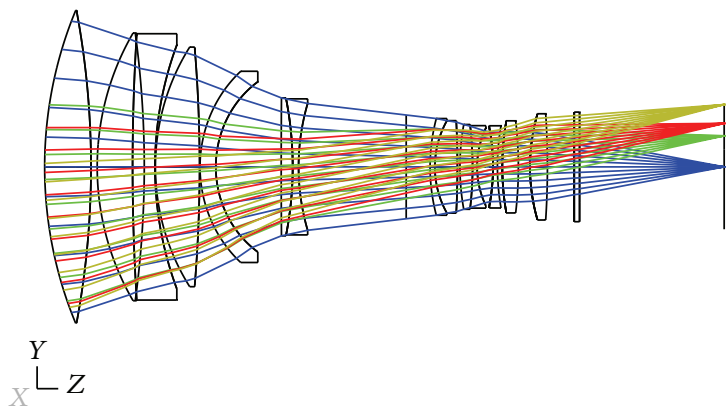

(a)

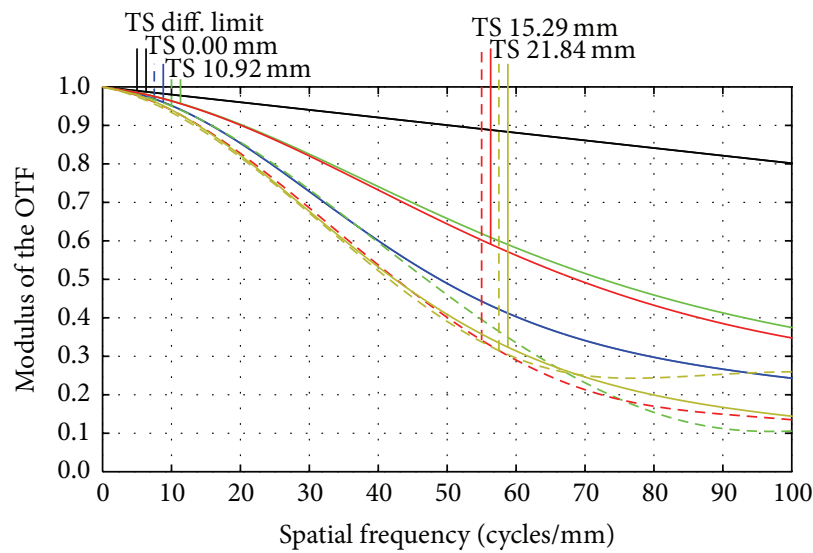

(c)

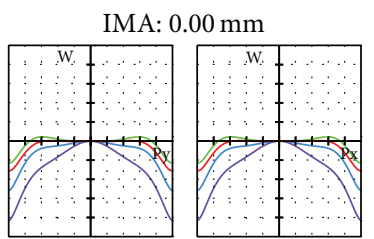

IMA: $15.29 \mathrm{~mm}$

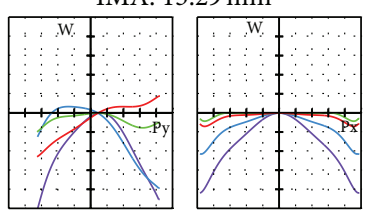

(e)

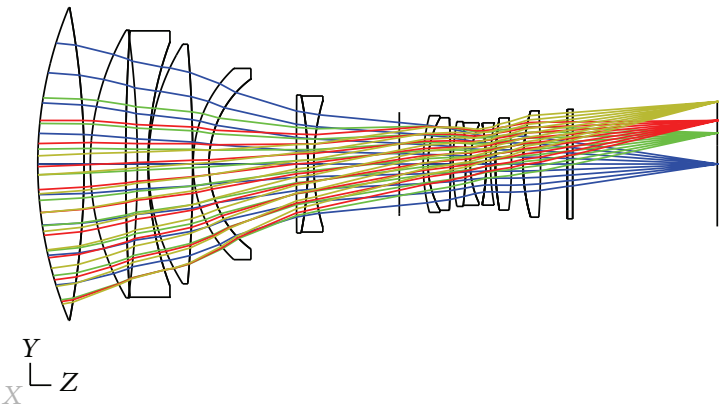

(b)

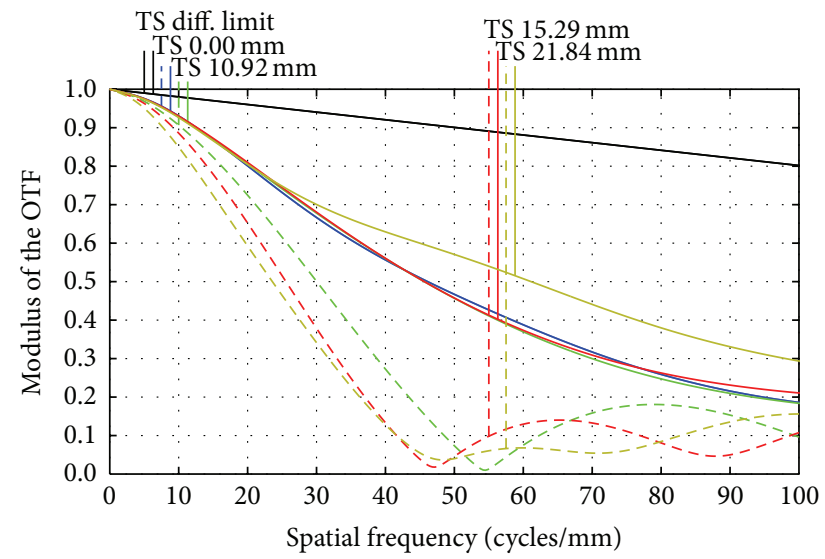

(d)
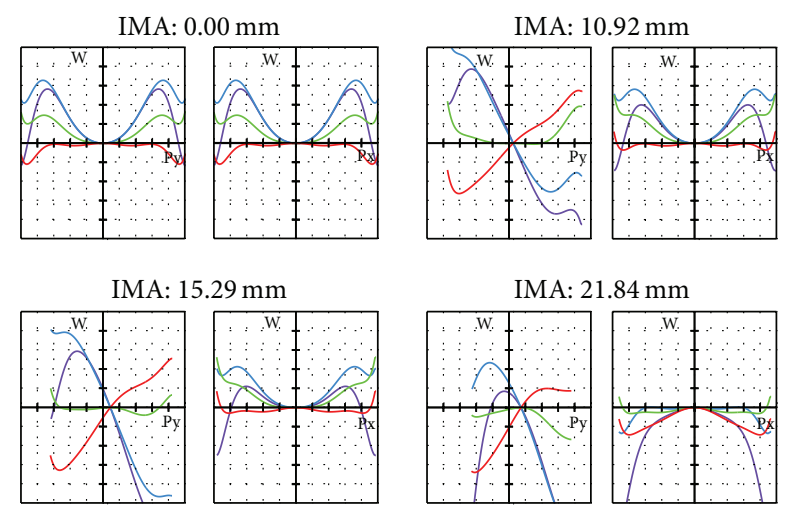

(f)

Figure 5: Canon telephoto lens original patent data for infinite ((a), (c), and (e)) and 2-meter ((b), (d), and (f)) object distance configurations. OPD fan scale is 2 waves. 


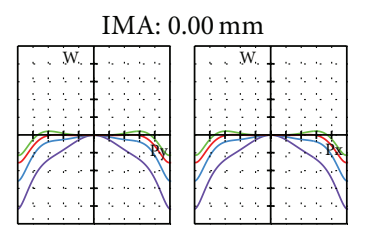

IMA: $15.29 \mathrm{~mm}$
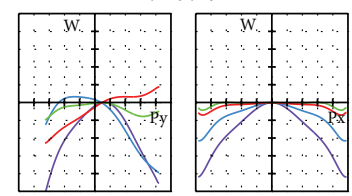

(a)

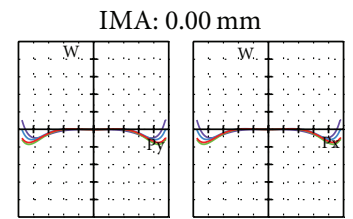

IMA: $15.29 \mathrm{~mm}$
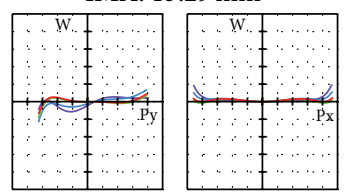

(c)

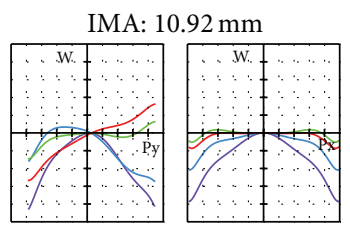

IMA: $21.84 \mathrm{~mm}$
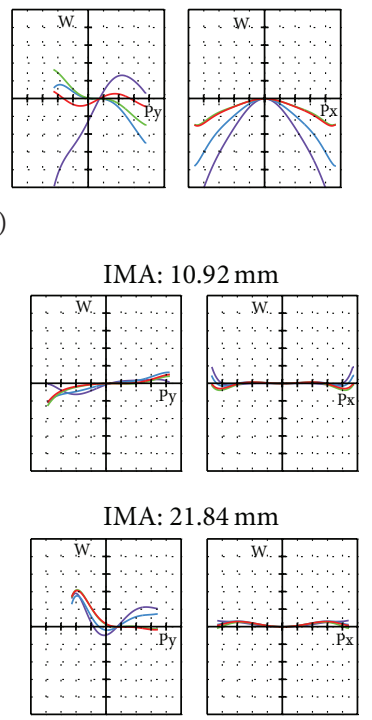

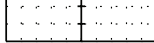
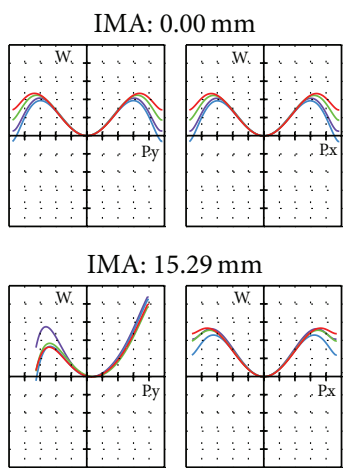
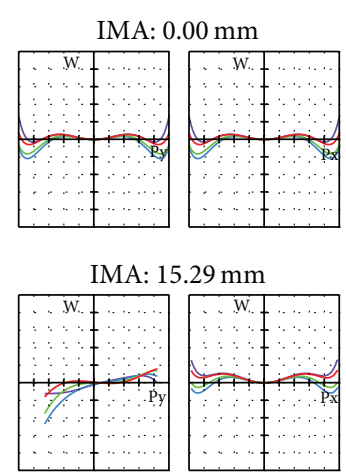

(d)

d)

FIGURE 6: OPD fans for infinite object distance: (a) patent data, (b) iteration 1, (c) iteration 2, and (d) iteration 3. Plot scale is 2 waves.
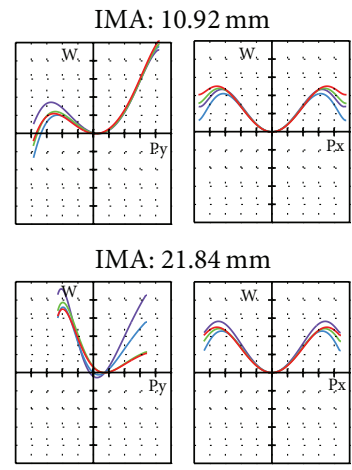

(b)
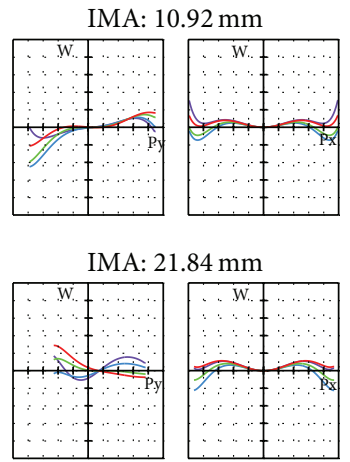
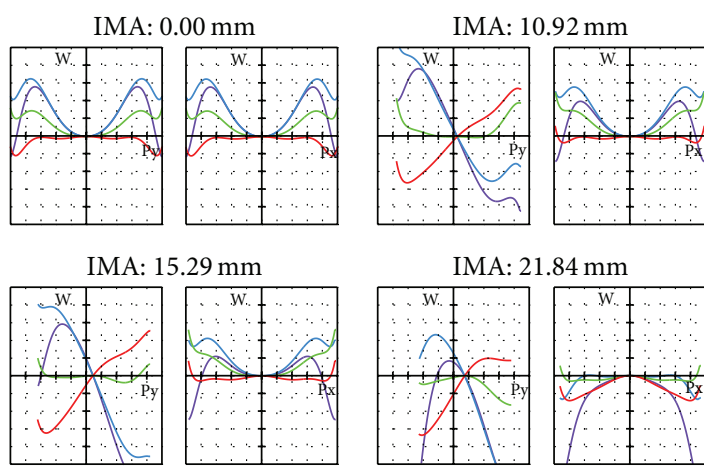

(a)

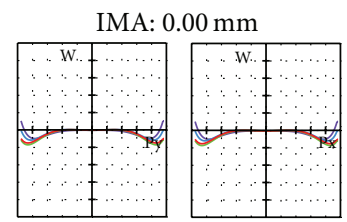

IMA: $15.29 \mathrm{~mm}$
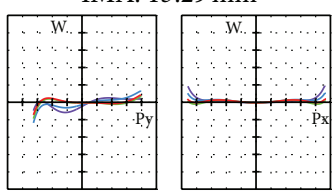

(c)

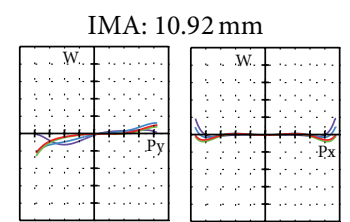

IMA: $21.84 \mathrm{~mm}$
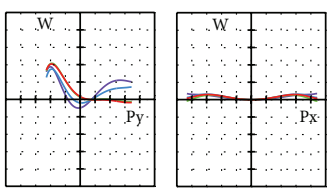
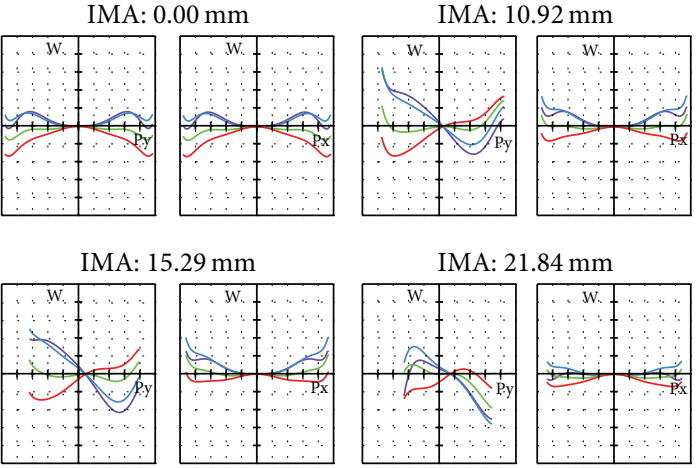

(b)
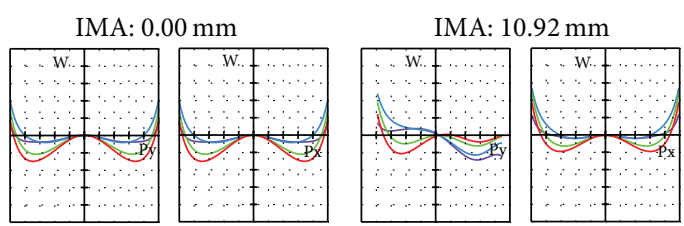

IMA: $21.84 \mathrm{~mm}$
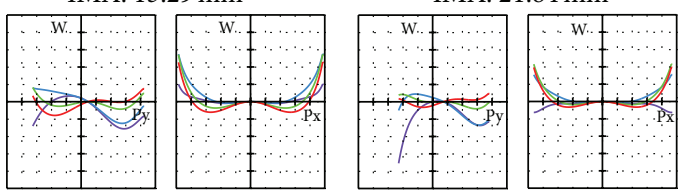

(d)

FIGURE 7: OPD fans for 2-meter object distance: (a) patent data, (b) iteration 1, (c) iteration 2, and (d) iteration 3. Plot scale is 2 waves. 


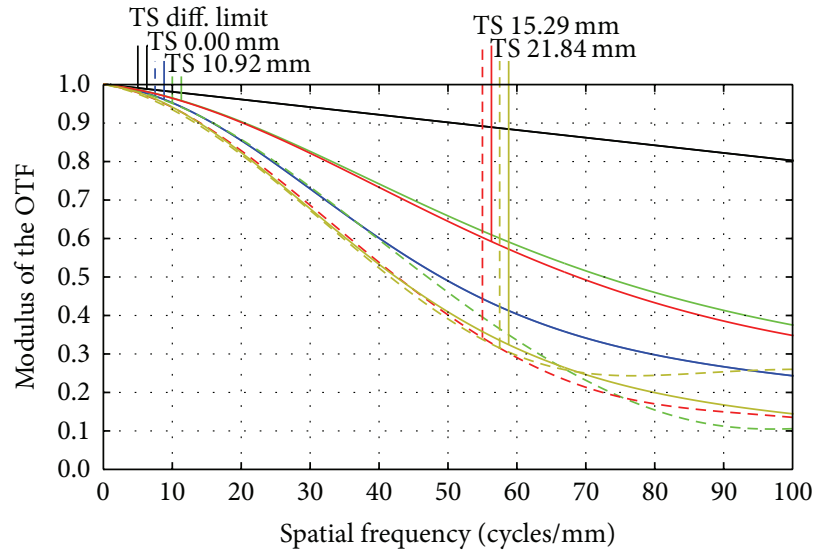

(a)

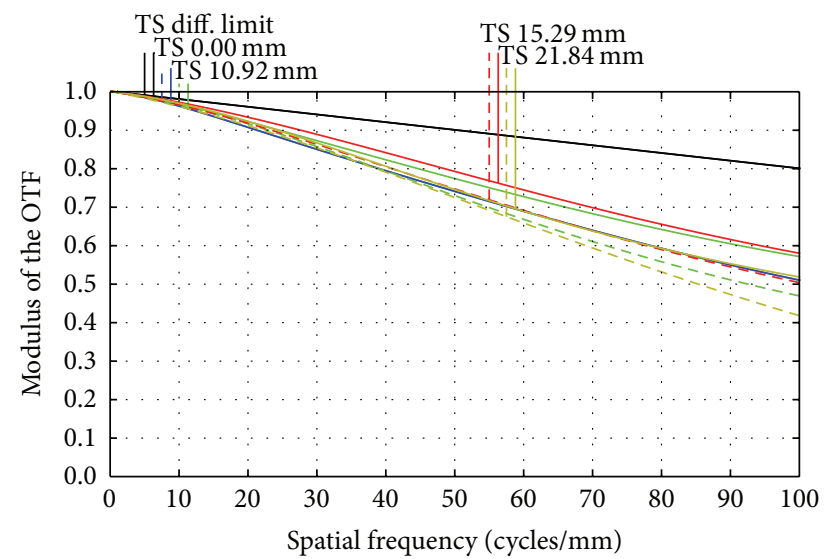

(b)

Figure 8: Polychromatic MTF for $436 \mathrm{~nm}$ to $656 \mathrm{~nm}$ for infinite object distance: (a) patent data and (b) final design.

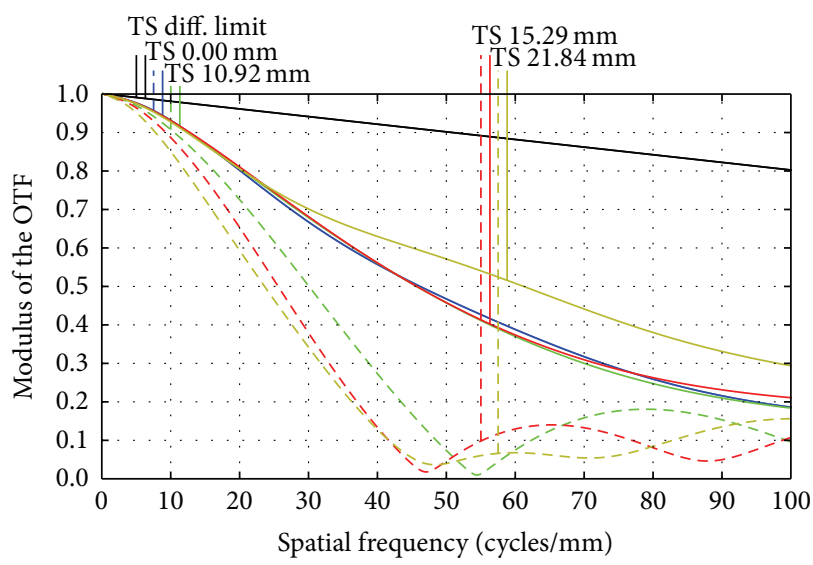

(a)

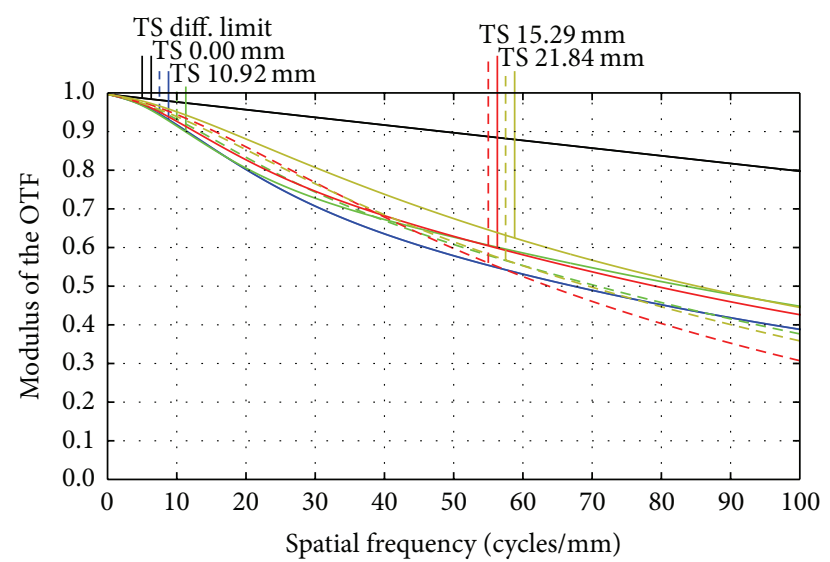

(b)

Figure 9: Polychromatic MTF from $436 \mathrm{~nm}$ to $656 \mathrm{~nm}$ for 2-meter object distance: (a) patent data and (b) final design.

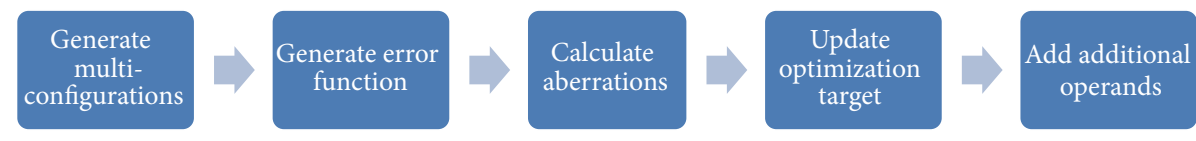

FIGURE 10: Aberration subtraction method for athermalization.

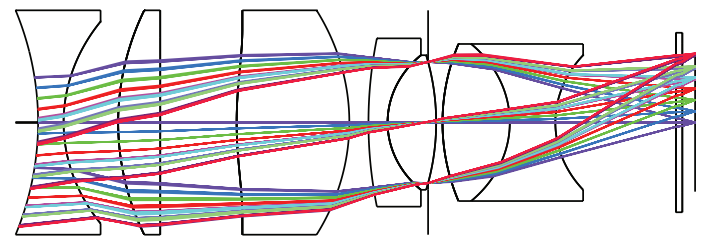

Y ᄂ Z

FIGURE 11: Single focus athermal lens.

targets are changed as follows. For any wavelength, field point, and pupil coordinate, we modify the wave or ray aberration target to be the aberration value at this point in the nominal configuration. The chief ray at the primary wavelength is used as a reference for both ray and wave aberrations calculations. Once again this procedure can easily be implemented by writing a macroprogram. Figure 10 shows the aberration subtraction procedure for athermalization.

We now comment on the dynamics of minimization of the modified error function. In the original design the nominal configuration's aberration will match the target aberration value, by construction. Initially the nominal configuration will not contribute to the error function. During the optimization, the original lens will change and the nominal configuration's aberration will be different. The weight placed on the nominal configuration, in the modified error function, will force the aberration correction to be close to the original design. Further minimization of the modified error function 

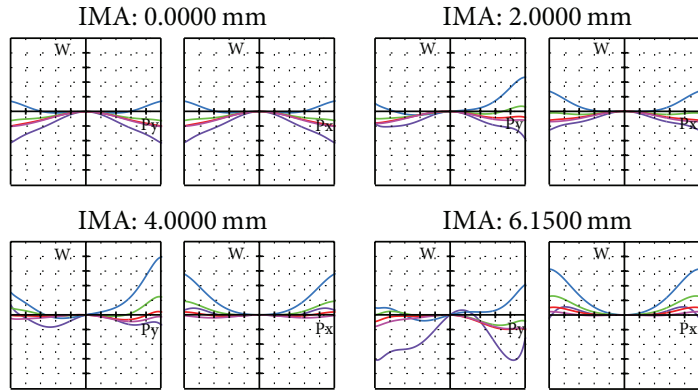

(a)

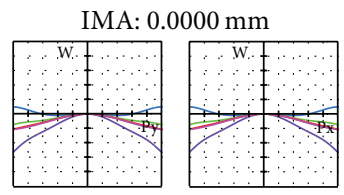

IMA: $4.0000 \mathrm{~mm}$
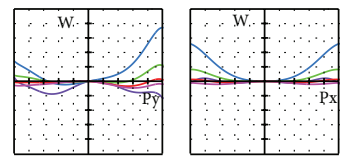

(c)

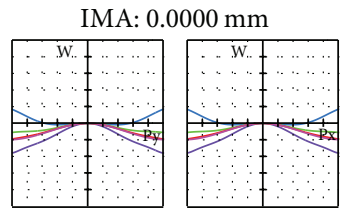

IMA: $4.0000 \mathrm{~mm}$
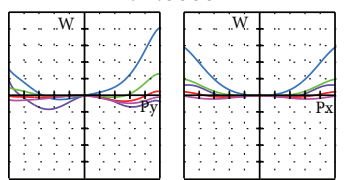

(e)

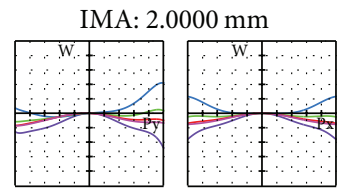

IMA: $6.1500 \mathrm{~mm}$
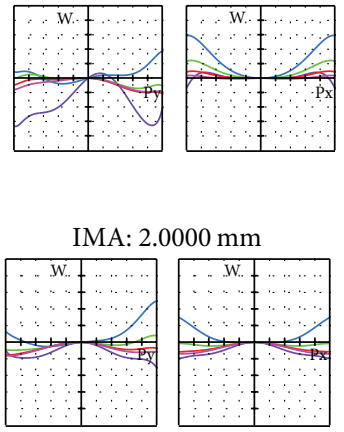

IMA: $6.1500 \mathrm{~mm}$
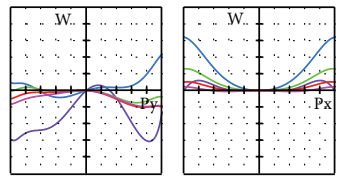

)

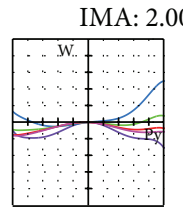

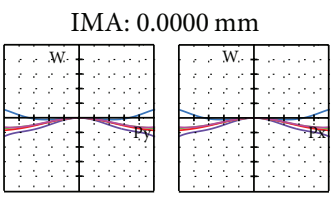
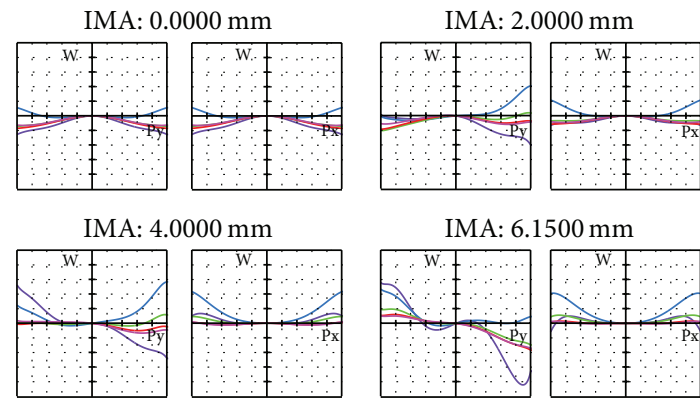

(b)
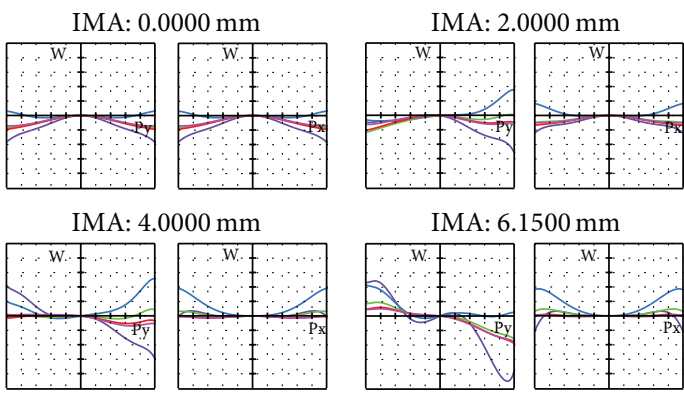

(d)

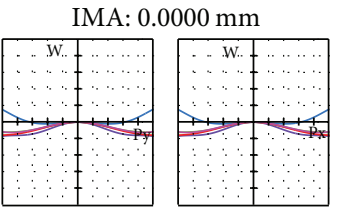

IMA: $4.0000 \mathrm{~mm}$
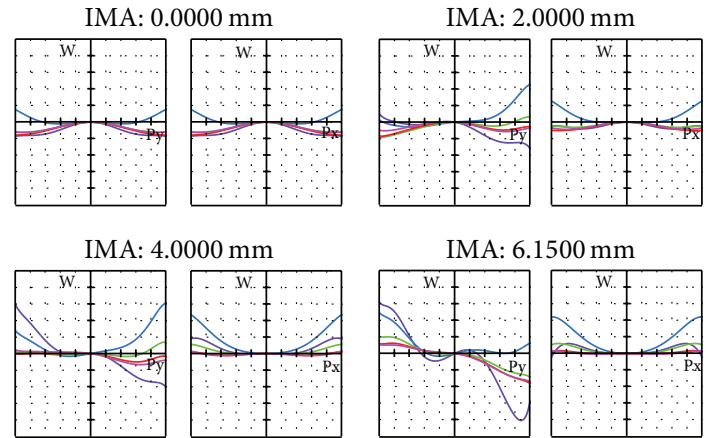

IMA: $6.1500 \mathrm{~mm}$

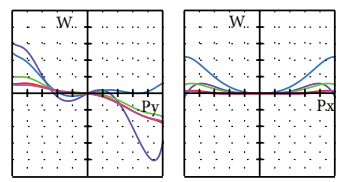

(f)

Figure 12: OPD fans: (a) original design at $20^{\circ} \mathrm{C}$, (b) final design at $20^{\circ} \mathrm{C}$, (c) original design at $-20^{\circ} \mathrm{C}$, (d) final design at $-20^{\circ} \mathrm{C}$, (e) original design at $60^{\circ} \mathrm{C}$, and (f) final design at $60^{\circ} \mathrm{C}$. Plot scale is 2 waves.

together with iterative substitution of similar glass types will reduce, if proper glass combinations exist, all other configuration's aberrations to the same nominal configuration's aberration value. Therefore a similar monochromatic and chromatic performance is maintained for the whole temperature range.

The aberrations induced by thermal effects are separated from the nominal design aberration and minimized to the same value. Similar to the algorithm described in Section 3, other optimization operands can be added to the modified error function to allow better control of various design parameters.

As an example we apply the presented method of athermalization to a single focus lens. This is a $14 \mathrm{~mm}$ focal length optical system that operates at $f / 1.75$. The lens supports a bandwidth from $420 \mathrm{~nm}$ in the visible to $1700 \mathrm{~nm}$ in the NIR. The lens is athermal from $-20^{\circ} \mathrm{C}$ to $+60^{\circ} \mathrm{C}$. An original system layout is shown in Figure 11.
We used the iteration algorithm described in Section 4. The OPD fans and MTF plots of the original design and final iteration are shown in Figures 12 and 13. The glass combinations used in the original design and final iteration are given in the Appendix.

This slight improvement of MTF over the entire field of view and the good balance was obtained for the whole temperature range.

\section{Conclusion}

We have presented two methods for chromatic aberration correction. Both methods are based on real ray tracing and can be implemented in commercial lens design software. The idea is to separate the chromatic aberration content from the monochromatic aberration content and use standard lens design software optimization tools, such as 


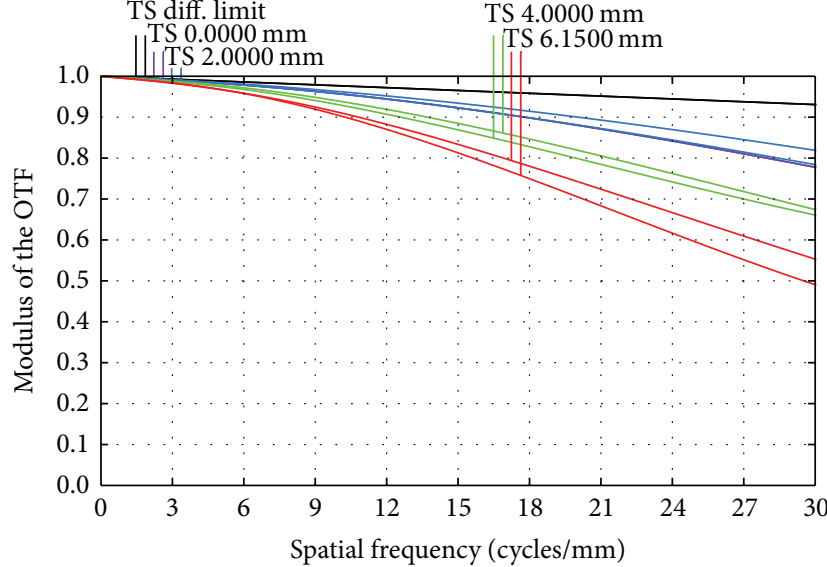

(a)

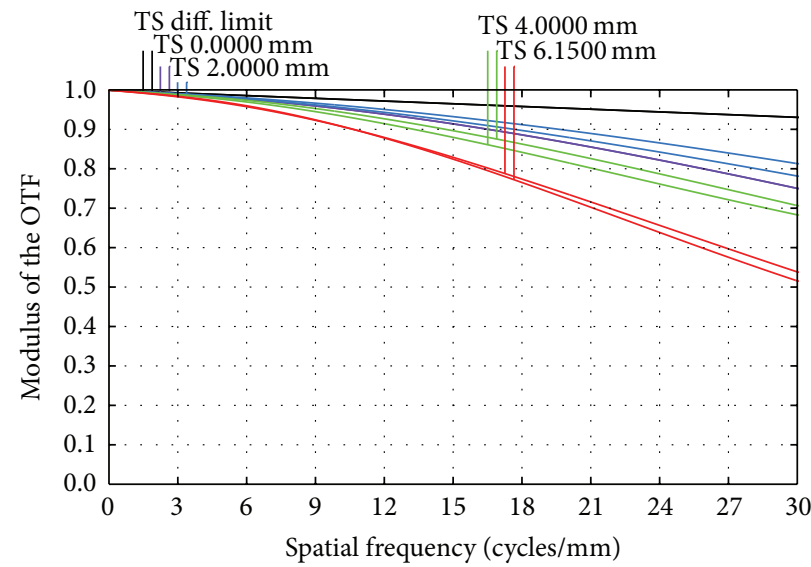

(c)

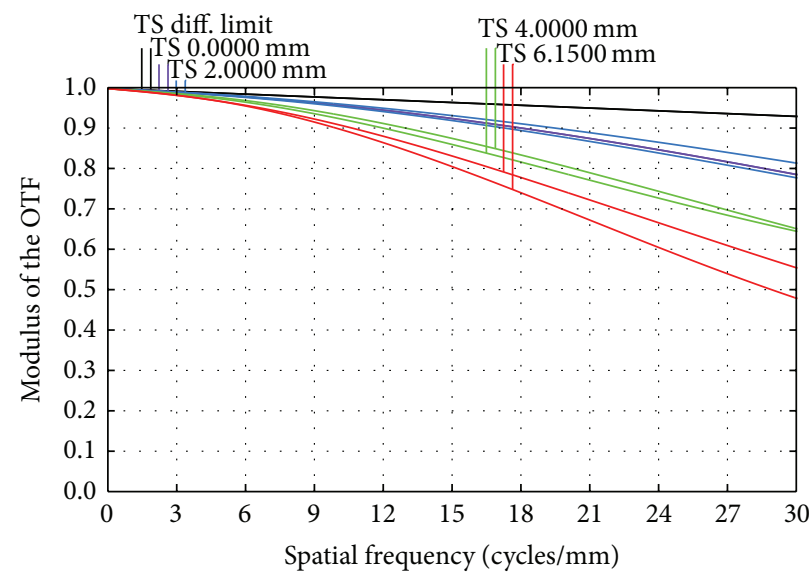

(e)

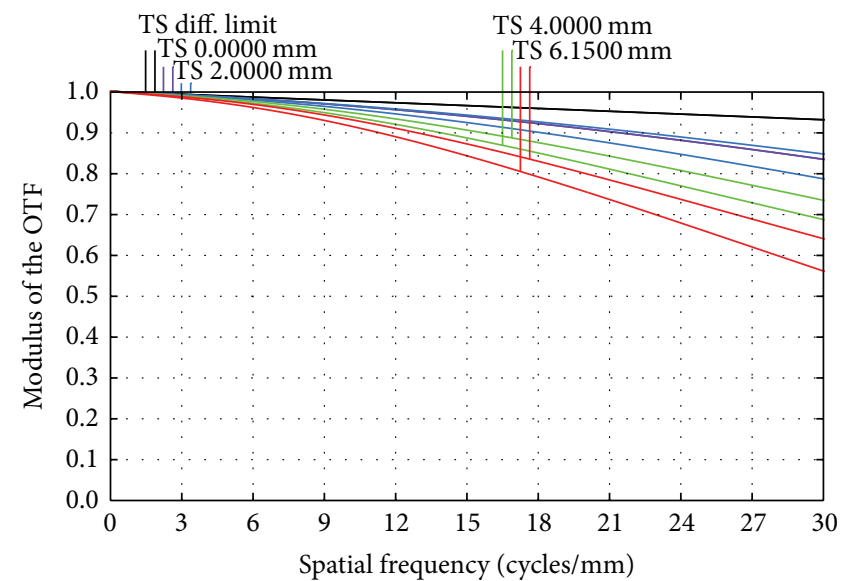

(b)

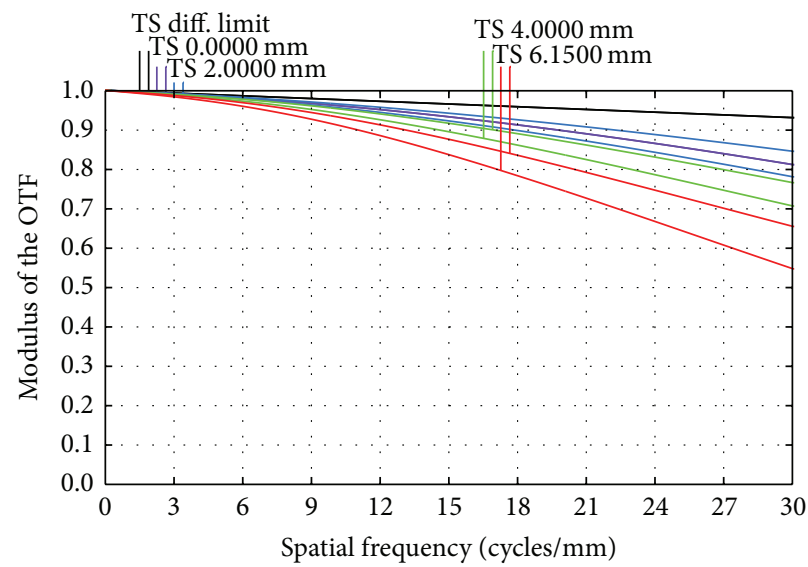

(d)

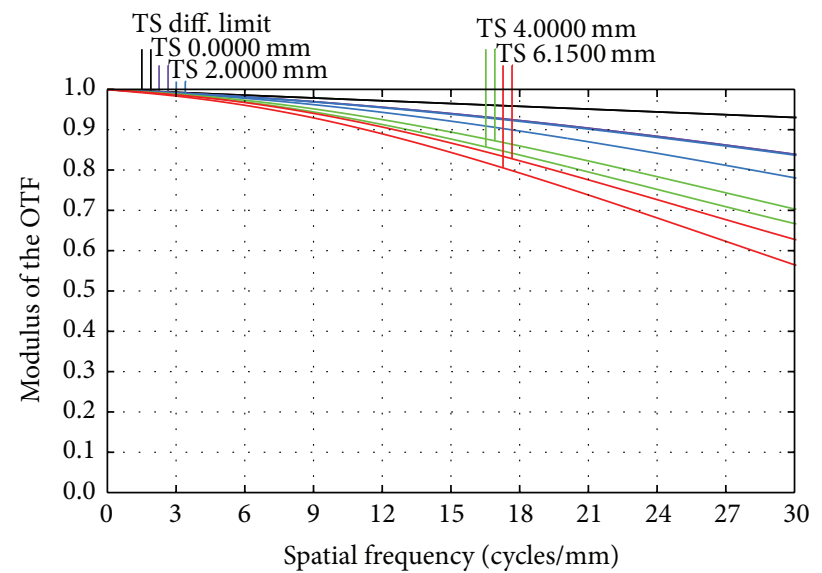

(f)

Figure 13: Polychromatic MTF for $420 \mathrm{~nm}$ to $1700 \mathrm{~nm}$ : (a) original design at $20^{\circ} \mathrm{C}$, (b) final design at $20^{\circ} \mathrm{C}$, (c) original design at $-20^{\circ} \mathrm{C}$, (d) final design at $-20^{\circ} \mathrm{C}$, (e) original design at $60^{\circ} \mathrm{C}$, and (f) final design at $60^{\circ} \mathrm{C}$.

global optimization and iterative glass substitution. The separation of aberrations removes the dependence in correction of one aberration type from the correction of other aberration types. In that case the optimization process is focused on reducing chromatic aberration to the monochromatic aberration value. We find these methods to be effective in correcting chromatic aberration beyond what a standard error function would do.

The presented methods are not limited to any specific bandwidth, can be used to correct both chromatic change of focus and chromatic change of magnification, support multiconfiguration systems, and do not require any 
TABLE 1: Optical glasses used in designs in Figures 6 and 7.

\begin{tabular}{|c|c|c|c|c|}
\hline Element number & Patent & Iteration 1 & Iteration 2 & Iteration 3 \\
\hline 1 & FCD1 & N-FK51A & PFK85 & LITHOTECH-CAF2 \\
\hline 2 & FCD1 & LITHOTECH-CAF2 & LITHOTECH-CAF2 & LITHOTECH-CAF2 \\
\hline 3 & S-LAM54 & N-LAF21 & LAFN21 & TAF4 \\
\hline 4 & LITHOTECH-CAF2 & LITHOTECH-CAF2 & LITHOTECH-CAF2 & LITHOTECH-CAF2 \\
\hline 5 & S-TIM1 & BAFD16 & PBM2 & F3 \\
\hline $6-1$ & S-TIH53 & S-NPH1 & S-NPH1 & SFL57 \\
\hline $6-2$ & S-LAH55V & TAF5 & YGH52 & YGH52 \\
\hline $7-1$ & S-NPH53 & S-NPH1 & S-NPH1 & S-NPH1 \\
\hline $7-2$ & S-LAL14 & LAKL12 & LACL1 & LAKN6 \\
\hline $8-1$ & S-TIH53 & $\mathrm{PBH} 23$ & FDS2 & SF10 \\
\hline $8-2$ & S-LAL7 & LAK12 & LAKN13 & LAFL1 \\
\hline 9 & S-LAH55V & S-LAM2 & SF9 & SF19 \\
\hline 10 & S-NSL3 & BSL7 & BAK2 & L-PHL2 \\
\hline 11 & S-BAL41 & P-SK57Q1 & PSK2 & BSC7 \\
\hline 12 & S-BSL7 & S-BSL7 & S-BSL7 & S-BSL7 \\
\hline
\end{tabular}

TABLe 2: Optical glasses used in designs in Figures 12 and 13.

\begin{tabular}{lcc}
\hline Element number & Original design & Final design \\
\hline 1 & N-SSK8 & K7 \\
2 & N-SK57 & N-SK57HT \\
3 & N-BAK2 & N-BAK2 \\
$4-1$ & N-SF57 & N-SK57HT \\
$4-2$ & N-FK51A & N-PK52A \\
$5-1$ & N-LASF40 & N-LASF40 \\
$5-2$ & N-KZFS2 & N-KZFS2 \\
\hline
\end{tabular}

additional/external calculations. Unlike other methods found in the literature, the presented methods are not limited to the finite number of aberration coefficients used in an error function. We use real ray tracing to reduce the chromatic aberrations. The user can take advantage of any additional lens design software features; for example, additional constraints can be added to the error function or a filter can be used to eliminate unwanted glass types.

We found that methods work the best when applied in the intermediate design stage, when monochromatic performance is reasonable and the chromatic aberrations are corrected to some extent.

As an example of application of the methods we reoptimize a Canon telephoto lens [9]. We looked for different glass combinations which would allow reducing chromatic aberrations while satisfying all other design constraints. We iterated the chromatic correction methods with regular optimization as described in Section 5 to gradually reduce the overall aberration in the system. The final glass combination, which was found by application of the aberration subtraction method, allows reducing both chromatic and monochromatic aberrations. Significant improvement in the polychromatic MTF over the entire FOV was obtained. The final solution is not readily found with standard optimization methods as can be tested.

Finally, we extended the methods to a different glass selection problem-athermalization of imaging systems. We modified the aberration subtraction algorithm to separate aberrations induced by temperature effects from the nominal design aberrations. In that case the optimization process is focused on reducing additional aberration due to thermal effects on the nominal aberration value.

Although the algorithms presented here are not limited to any specific lens design software, in this work we used Radiant-Zemax lens design program. The pupil was sampled using Gaussian quadrature method with 20 rings and 12 arms. Optimization time, which of course depends on the complexity of particular lens, is typically less than one hour for a simple system.

\section{Appendix}

Optical glasses used in designs shown in Figures 6 and 7 are presented in Table 1.

Optical glasses used in designs shown in Figures 12 and 13 are presented in Table 2 .

\section{Conflict of Interests}

The authors declare that there is no conflict of interests regarding the publication of this paper.

\section{Acknowledgment}

This work has been sponsored by Canon USA. 


\section{References}

[1] J. L. Rayces and M. Rosete-Aguilar, "Differential equation for normal glass dispersion and evaluation of the secondary spectrum," Applied Optics, vol. 38, no. 10, pp. 2028-2039, 1999.

[2] J. L. Rayces and M. Rosete-Aguilar, "Selection of glasses for achromatic doublets with reduced secondary spectrum. I. Tolerance conditions for secondary spectrum, spherochromatism, and fifth-order spherical aberration," Applied Optics, vol. 40, no. 31, pp. 5663-5676, 2001.

[3] P. N. Robb, "Selection of optical glasses. 1: two materials," Applied Optics, vol. 24, no. 12, pp. 1864-1877, 1985.

[4] R. I. Mercado and P. N. Robb, "Color-corrected optical systems and method of selecting optical materials therefor," U.S Patent 5210646A, 1993.

[5] B. F. C. Albuquerque, J. Sasian, F. L. Sousa, and A. S. Montes, "Method of glass selection for color correction in optical system design," Optics Express, vol. 20, pp. 13592-13611, 2012.

[6] R. D. Sigler, "Glass selection for airspaced apochromats using the Buchdahl dispersion equation," Applied Optics, vol. 25, pp. 4311-4320, 1986.

[7] J. M. Sasián, S. A. Lerner, T. Y. Lin, and L. Laughlin, "Ray and van Cittert-Zernike characterization of spatial coherence," Applied Optics, vol. 40, no. 7, pp. 1037-1043, 2001.

[8] R. N. Pfisterer, "Clever tricks in optical engineering," in 7 th Novel Optical Systems Design and Optimization, pp. 230-239, Denver, Colo, USA, August 2004.

[9] M. Misaka, A. Nishio, and H. Ogawa, "Optical system and optical apparatus having the same," U.S. Patent 6,115,188A, 2000. 

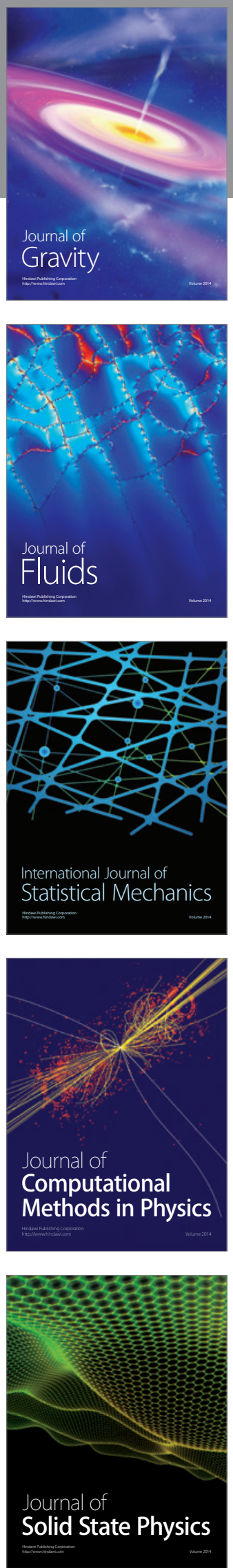

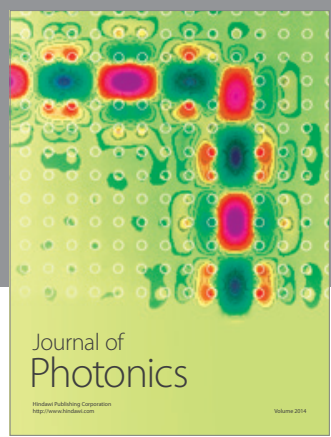

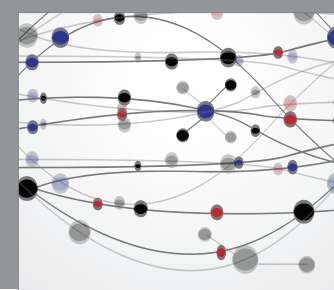

The Scientific World Journal

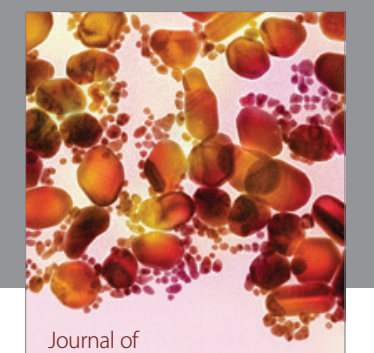

Soft Matter
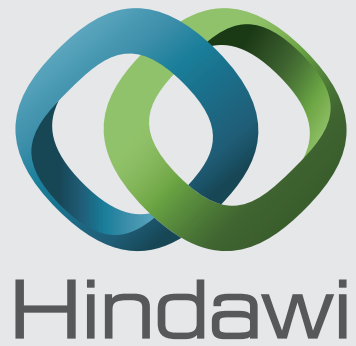

Submit your manuscripts at

http://www.hindawi.com
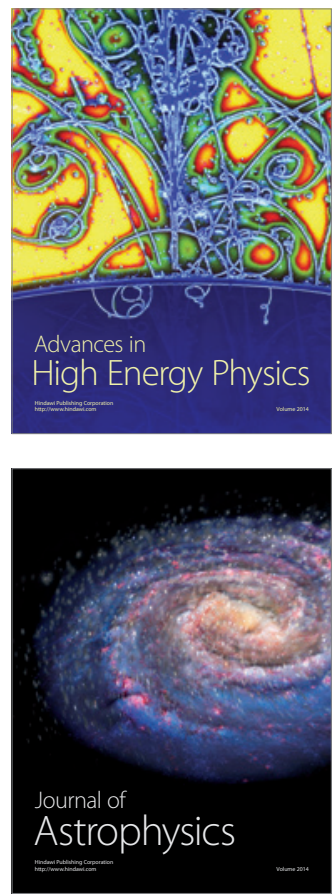
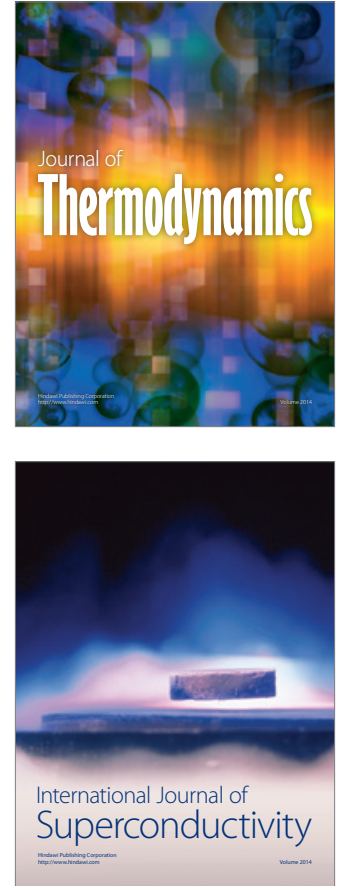
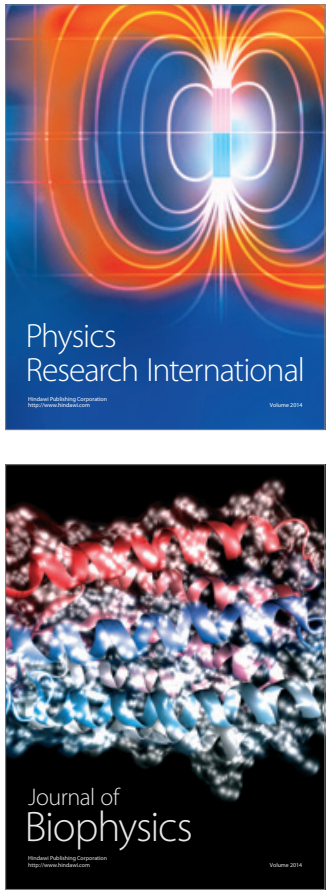
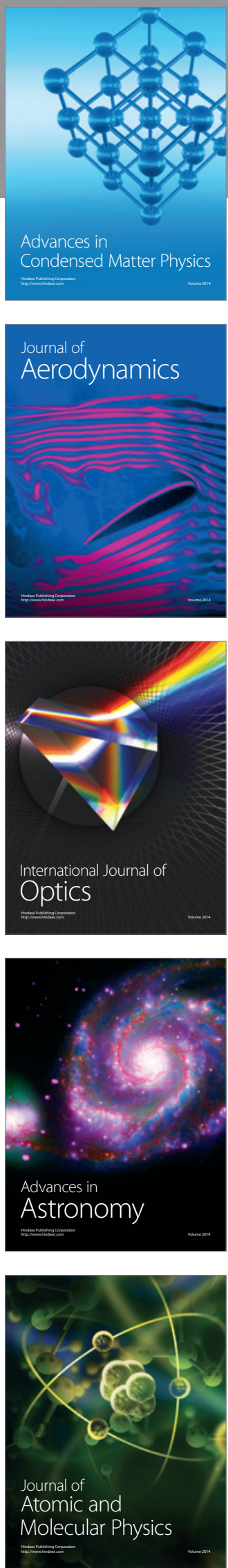